\title{
Panax notoginseng Promotes Repair of Colonic Microvascular Injury in Sprague-Dawley Rats with Experimental Colitis
}

\author{
Shiying Wang, ${ }^{1,2}$ Ping Tao, ${ }^{1}$ Lei Zhao, ${ }^{1}$ Wangjun Zhang, ${ }^{1}$ Hongyi Hu $\mathbb{D},{ }^{1,2}$ and Jiang Lin $\mathbb{D}^{1}$ \\ ${ }^{1}$ Department of Gastroenterology, Longhua Hospital, Shanghai University of TCM, Shanghai, China \\ ${ }^{2}$ Institute of Digestive Diseases, China-Canada Center of Research for Digestive Diseases (ccCRDD), Longhua Hospital, \\ Shanghai University of TCM, Shanghai, China
}

Correspondence should be addressed to Hongyi Hu; hongyihu2003@shutcm.edu.cn and Jiang Lin; linjiang@longhua.net

Received 10 November 2017; Revised 20 December 2017; Accepted 27 December 2017; Published 29 January 2018

Academic Editor: Yuan Xu

Copyright (C) 2018 Shiying Wang et al. This is an open access article distributed under the Creative Commons Attribution License, which permits unrestricted use, distribution, and reproduction in any medium, provided the original work is properly cited.

\begin{abstract}
To investigate the therapeutic effects of PN on intestinal inflammation and microvascular injury and its mechanisms, dextran sodium sulfate- (DSS-) or iodoacetamide- (IA-) induced rat colitis models were used. After colitis model was established, PN was orally administered for 7 days at daily dosage of $1.0 \mathrm{~g} / \mathrm{kg}$. Obvious colonic inflammation and mucosal injuries and microvessels were observed in DSS- and IA-induced colitis groups. DAI scores, serum concentrations of VEGFA121, VEGFA165, VEGFA165/VEGFA121, IL-6, and TNF- $\alpha$, and expression of Rap1GAP and TSP1 proteins in the colon were significantly higher while serum concentrations of IL-4 and IL-10 and MVD in colon were significantly lower in the colitis model groups than in the normal control group. PN promoted repair of colonic mucosal injury and microvessels, attenuated inflammation, and decreased DAI scores in rats with colitis. PN also decreased the serum concentrations of VEGFA121, VEGFA165, VEGFA165/VEGFA121, IL-6, and TNF- $\alpha$ and increased the serum concentrations of IL-4 and IL-10, with the expression of Rap1GAP and TSP1 proteins in colonic mucosa being downregulated. The constituents of PN were identified with HPLC-DAD. To sum up, PN could promote repair of injuries of colonic mucosa and microvessels via downregulating VEGFA isoforms and inhibiting Rap1GAP/TSP1 signaling pathway.
\end{abstract}

\section{Introduction}

Colonic mucosal ulcers and increased intestinal permeability are the major pathological features of UC [1]. Colonic microvascular injury has been widely observed in UC patients and considered as an important pathogenesis of UC in recent years [2]. Mucosal microvessels injury accompanied with increased vascular permeability $[3,4]$ results in the imbalance between oxygen consumption and oxygen delivery in colonic mucosa [5]. In addition, our previous study found that colonic mucosal damage was significantly improved when mucosal microvessels injury was repaired in rats with experimental colitis [6]. Therefore, we speculate that microvascular injury may play an important role in the pathogenesis of UC and may be a new target in UC treatment.

Panax notoginseng (PN), a traditional Chinese medicine used to treat vascular lesions, was found to promote repair of colonic mucosal damage in UC $[7,8]$. PN has various bioactive components. Some of them, such as Notoginsenoside R1 and Ginsenoside Rgl, promote growth and proliferation of vascular endothelial cell (VEC) and play an important role in maintaining the structure and the function of blood vessels $[9,10]$. Although PN has been widely used to treat UC in recent years and has an obvious effect on repairing colonic mucosa damage [8], its mechanism is still unclear Therefore, we hypothesized that PN could promote repair of mucosal damage via regulating angiogenesis.

\section{Materials and Methods}

2.1. Animals. Sprague-Dawley (SD) rats $(120 \sim 140 \mathrm{~g})$ were purchased from Shanghai SLAC Laboratory Animal, Co., Ltd. (Shanghai, China). The Institutional Animal Care and Use Committee of Shanghai University of Traditional Chinese Medicine approved all procedures (number SZY201612006). Rats were housed in a pathogen-free environment and allowed to acclimate to the environment for $7 \mathrm{~d}$ before inclusion in an experiment. 
2.2. Preparation of $P N$. PN was purchased from Shanghai Huayu Traditional Chinese Medicine Co., Ltd. (Shanghai, China). PN $(0.9 \mathrm{~kg})$ was dispersed in tap water $(6 \mathrm{~L})$, boiled at $100^{\circ} \mathrm{C}$ for $3 \mathrm{~h}$, filtered through a sieve $(150 \mu \mathrm{m})$, extracted with absolute ethanol, and dried in a freeze dryer. The browncolored extract powder of $\mathrm{PN}$ was stored at $-20^{\circ} \mathrm{C}$.

2.3. Identification of Phytochemical Constituents. A Shimadzu LC system (Shimadzu, Kyoto, Japan) was employed for liquid chromatographic analysis. The LC system was connected by a hybrid ion trap/time-of-flight mass spectrometer equipped with an electrospray ionization (ESI) source to perform highresolution tandem mass spectrometry. HPLC analysis was conducted on a Jade-Pak ODS-AQ column $(250 \times 4.6 \mathrm{~mm}$, $5 \mu \mathrm{m}$, TechWay). The mobile phase $(1.0 \mathrm{~mL} / \mathrm{min})$ consists of aqueous acetonitrile (A) $-0.1 \%$ - with formic acid (B) with a gradient program as follows: $0-27 \mathrm{~min}, 18.5 \%-23 \% \mathrm{~A}$; 27-50 min, 23\%-21\% A; 50-53 min, 21\%-29\% A; 53-110 min, $29 \%-37 \%$ A. Roughly $25 \%$ portion of the effluent was introduced into the ESI source by splitting the effluent via two PEEK tubes with length ratio of $1: 3$. A detection wavelength of $203 \mathrm{~nm}$ and an injection volume of $10 \mu \mathrm{L}$ were set. Optimization of the operating conditions and the equipment and the reagents for HRMS analysis were identical to those used in the previous study [11].

2.4. IA- and DSS-Induced Colitis. Two rat models of colitis were used in this study. One was induced by $0.1 \mathrm{~mL} 6 \%$ IA (Sigma-Aldrich, MO, USA) dissolved in 1\% methylcellulose given to rats once by enema ( $7 \mathrm{~cm}$ above the anus) via rubber catheter Nelaton S-8 (Rüsch, Germany). The other was induced by DSS (Sigma-Aldrich, MO, USA): rats were allowed free access to purified water containing 5\% DSS $(\mathrm{w} / \mathrm{v})$ for $7 \mathrm{~d}$. DSS solution was prepared daily as previously reported [12].

2.5. PN Intervention In Vivo. SD rats were divided into normal control group $(n=6)$, experimental colitis model control group $(n=6)$, and $\mathrm{PN}$ intervention group $(n=$ $6)$. For the intervention groups, $P N$ was intragastrically administered to rats with IA-induced or DSS-induced colitis once daily for seven consecutive days at the dosage of $1.0 \mathrm{~g} / \mathrm{kg}$. For the control groups, normal saline was given to the corresponding rats. The initial administration times of $\mathrm{PN}$ were $3 \mathrm{~d}$ after establishing the colitis models. After 7 days of treatment, the rats were sacrificed for pathological evaluation, vascular permeability analysis, and cytokines detection. Three independent experiments were performed in triplicate.

2.6. Disease Activity Index (DAI). The severity of colitis was evaluated with DAI, as described previously [13]. The parameters of DAI included weight loss (0, none; $1,0-5 \% ; 2$, $5-10 \% ; 3,10-20 \% ; 4,>20 \%$ ); stool consistency ( 0 , none; 2 , loose stool; 4 , watery); and bleeding ( 0 , none; 1 , trace; 2 , mild occult blood; 3 , obvious occult blood; 4 , gross bleeding). DAI was scored before, during, and after the treatment.

2.7. Histological Assay. Segments of colon were fixed in formalin buffer and embedded in paraffin. Sections $5 \mu \mathrm{m}$ thick were deparaffinized and stained with hematoxylin and eosin $(\mathrm{H} \& \mathrm{E})$. The histological changes were assessed by a pathologist.

2.8. Microvessel Density (MVD) Analysis. $5 \mu \mathrm{m}$ thick paraffin-embedded sections of colon were deparaffinized, subjected to heat-mediated antigen retrieval, and blocked with goat serum. The tissues were incubated with the primary anti-CD31 antibody (1:200, v/v) (Abcam, UK) overnight at $4^{\circ} \mathrm{C}$. After three $5 \mathrm{~min}$ washes, the horseradish peroxidase(HRP-) labeled secondary antibody $(1: 300)$ was added and the samples were incubated at $37^{\circ} \mathrm{C}$ for $1 \mathrm{~h}$. The sections were counterstained with hematoxylin for $1 \mathrm{~min}$ at room temperature to visualize the endothelial cell nuclei. Three fields with CD31-positive cells in each section were chosen to assess MVD. The MVD value was calculated as the average vessel counts in three selected areas within a microscopic field.

2.9. Vascular Permeability (VP) in Rats. VP of vessels in colonic mucosa was evaluated by the Evans blue method, as described previously [14]. Rats were anesthetized with intraperitoneal injection of sodium pentobarbital. Evans blue $(1 \mathrm{mg} / 100 \mathrm{~g})$ (Sigma-Aldrich, MO, USA) was injected intravenously $15 \mathrm{~min}$ before autopsy. Evans blue was extracted from the $1 \mathrm{~cm}$ segment of colonic tissue using formamide and measured by spectrophotometry at $610 \mathrm{~nm}$. Results were expressed as OD value per milligram of colon.

2.10. Enzyme-Linked Immunosorbent Assay (ELISA). Blood samples were collected from the abdominal aorta. The serum concentrations of VEGFA165, VEGFA121 (CloudClone Corp, TX, USA), IL-4 (R\&D, MN, USA), IL-6 (R\&D, MN, USA), IL-10 (R\&D, MN, USA), and TNF- $\alpha$ (R\&D, MN, USA) were detected using the appropriate ELISA kits. Absorbance was measured on a microtitre plate reader.

2.11. Western Blotting. Rap1GAP and TSP1 proteins were measured by western blotting as described previously [6]. Colonic tissue was cut into pieces and homogenized in fivefold volumes of ice-cold homogenizing buffer $(0.1 \mathrm{mmol} / \mathrm{L}$ $\mathrm{NaCl}, 0.1 \mathrm{~mol} / \mathrm{L}$ Tris- $\mathrm{HCl}$, and $0.001 \mathrm{~mol} / \mathrm{L}$ EDTA) containing $1 \mathrm{mmol} / \mathrm{L}$ phenylmethylsulfonyl fluoride, $1 \mathrm{mg} / \mathrm{mL}$ aprotinin, and $0.1 \mathrm{mmol} / \mathrm{L}$ leupeptin at $3000 \times \mathrm{g}$ and $4^{\circ} \mathrm{C}$ for $1 \mathrm{~h}$. Bovine serum albumin was used to estimate the protein content in supernatants. The protein samples $(60 \mu \mathrm{g}$ in each sample) were subjected to SDS-PAGE and transferred to polyvinylidene fluoride membranes using a transfer apparatus (Bio-Rad, Hercules, CA, USA). The membranes were blocked for $2 \mathrm{~h}$; then the primary antibodies anti-Rap1GAP $(1: 1000)$ and anti-TSP1 $(1: 1000)$ were added and incubated at $4^{\circ} \mathrm{C}$ overnight, and the corresponding HRP-conjugated secondary antibody (Cell Signaling Technology) was added and incubated for $1 \mathrm{~h}$. Protein-antibody complexes were detected by Clarity Western ECL Substrate (Bio-Rad), and results were authenticated with the Image J software (Gene Co., Ltd., China).

2.12. Statistical Analysis. Data were presented as the mean \pm $\mathrm{SD}$. One-way ANOVA or general linear model with repeated 


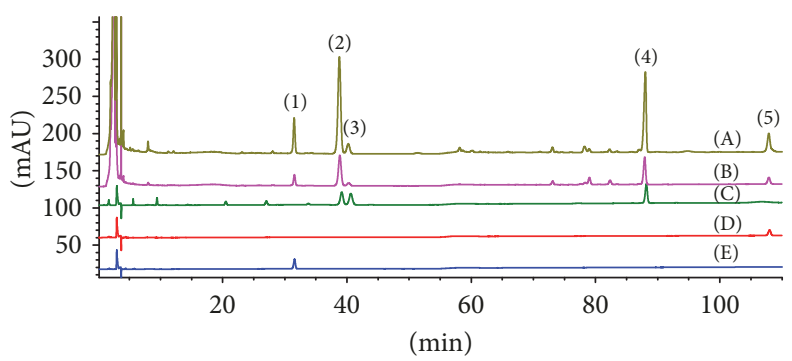

FIGURE 1: The HPLC analysis of the extract of Panax notoginseng. (A) Exact of PN; (B) notoginseng formula particles (allowed for using in clinic, Lot: A1500376); (C) standards sample 1* (Ginsenoside Rg1, Ginsenoside Re, and Ginsenoside Rb1); (D) standards sample 2 (Ginsenoside Rd); (E) standards sample 3 (Notoginsenoside R1). Notoginsenoside R1 (1) was detected at approximately 33.001 minutes, Ginsenoside Rg1 (2) was detected at approximately 39.175 minutes, Ginsenoside Re (3) was detected at approximately 40.632 minutes, Ginsenoside Rb1 (4) was detected at approximately 88.182 minutes, and Ginsenoside Rd (5) was detected at approximately 107.994 minutes. ${ }^{*}$ They are hard to be completely purified.

measures was used to analyze the data sets with three or more groups and least significant difference post hoc test for multiple comparisons. Student's $t$-test was used to analyze data sets with two groups. $P<0.05$ was considered significant.

\section{Results}

3.1. The Main Phytochemical Constituents in the PN. Five saponins were found in $\mathrm{PN}$ via comparing the retention times of the isolated compounds in HPLC chromatogram and analyzing MZ data. These five saponins were Notoginsenoside R1 (0.094 mg/mL, retention time (RT): $31.571 \mathrm{~min})$, Ginsenoside Rg1 (0.15264 mg/mL, RT: $39.175 \mathrm{~min})$, Ginsenoside Re (0.17744 mg/mL, RT: $40.632 \mathrm{~min})$, Ginsenoside Rb1 (0.24384 mg/mL, RT: $88.182 \mathrm{~min}$ ), and Ginsenoside Rd (0.11857 mg/mL, RT: $107.994 \mathrm{~min})$, respectively (Figure 1). The structures of these five saponins were described in previous study [4].

3.2. The Effects of PN on Colonic Mucosal Inflammation. Compared with the normal control group, DAI scores were much higher in the DSS- and IA-induced experimental colitis control groups (Figure 2(b)). Obvious hyperemia and inflammatory cells infiltration were found in the colitis control groups (Figures 2(c) and 2(d)) accompanied with higher proinflammatory cytokines (IL- 6 and TNF- $\alpha$ ) and lower antiinflammatory cytokines (IL-4 and IL-10) (Figure 2(e)). After being treated by PN, DAI scores were decreased, hyperemia and inflammatory cells infiltration were attenuated, proinflammatory cytokines were downregulated, and antiinflammatory cytokines were upregulated in the rats with colitis. These results suggested that PN could attenuate the inflammation and improve repair of mucosal injury induced by DSS or IA.

3.3. The Effects of PN on Mucosal Microvascular Injury and Vascular Permeability. Compared with the normal control group, the MVD values were significantly decreased and vascular permeability was significantly increased in the colitis control groups (Figures 2(f) and 3(a)). However, after treatment by PN, the MVD values were increased while the vascular permeability was decreased in the rats with colitis. These results indicated that $\mathrm{PN}$ might improve repair of mucosal microvascular injury.

3.4. PN Reversed the Disordered Serum Ratio of VEGFA165/ VEGFA121. The serum concentrations of VEGFA165 and VEGFA121 and the serum ratio of VEGFA165/VEGFA121 were significantly increased in IA- and DSS-induced experimental colitis groups compared with the normal control group. After treatment by $\mathrm{PN}$, the increased serum concentrations of VEGFA165 and VEGFA121 were downregulated and the disordered serum ratio of VEGFA165/VEGFA121 was reversed in the rats with colitis (Figures 3(b) and 3(c)).

3.5. PN Inhibited Rap1GAP/TSP1 Signaling Pathway. The expression of Rap1GAP and TSP1 proteins was significantly increased in IA- and DSS-induced experimental colitis groups compared with the normal control group. After treatment by PN, the expression of Rap1GAP and TSP1 proteins in colonic mucosa was reversed in the rats with colitis (Figures 4(a) and 4(b)).

\section{Discussion}

$\mathrm{PN}$ is a Chinese herbal medicine with multiple pharmacological effects and widely used to treat cardiovascular diseases, pain, inflammation, and hemorrhagic injuries [15]. The bioactive components and their contents in PN are different depending on the place of origin and the season of collection. In order to evaluate the quality of $\mathrm{PN}$, we used high performance liquid chromatography (HPLC) to analyze the composition of PN used in this study. The main components of PN in this study included Notoginsenoside R1, Ginsenoside Rg1, Ginsenoside Re, Ginsenoside Rb1, and Ginsenoside $\mathrm{Rd}$. The components and their contents are in line with the provisions of the Chinese Pharmacopoeia.

It was found that significant microvascular injury occurred in the early stage of UC onset and it might be important for promoting the development of UC [3]. The oxygen supply in colonic mucosa is dependent on the number 


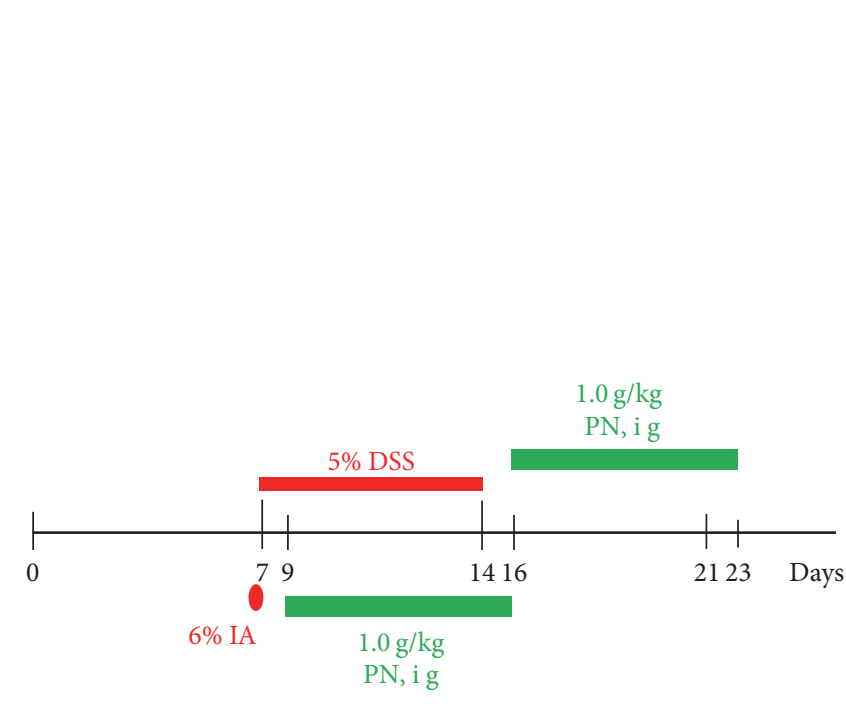

(a)

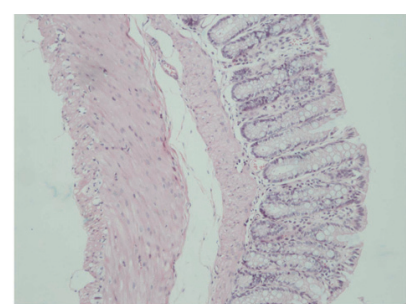

NS

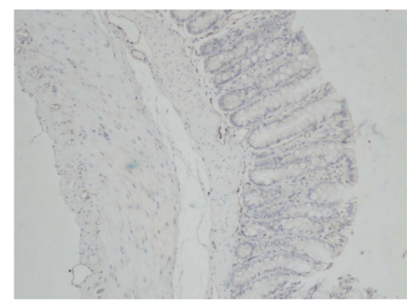

NS

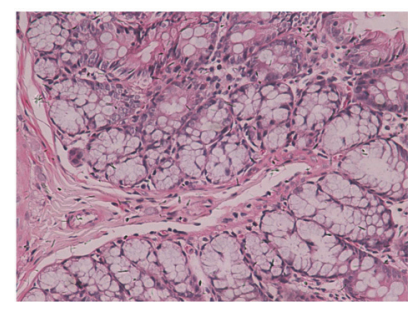

NS

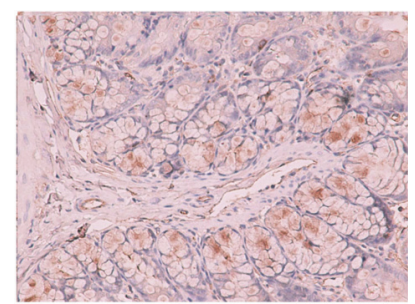

NS

IA

IA

(c)

DSS

DSS

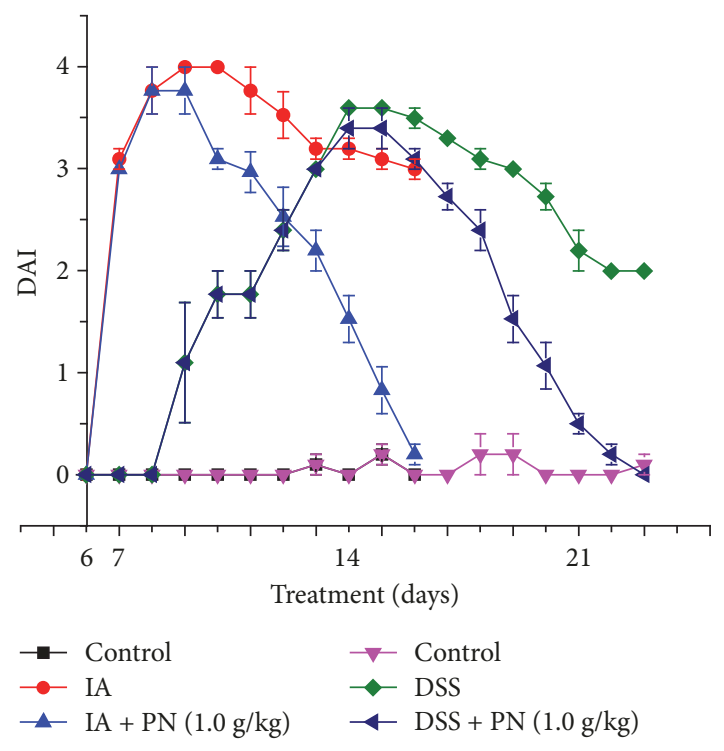

(b)
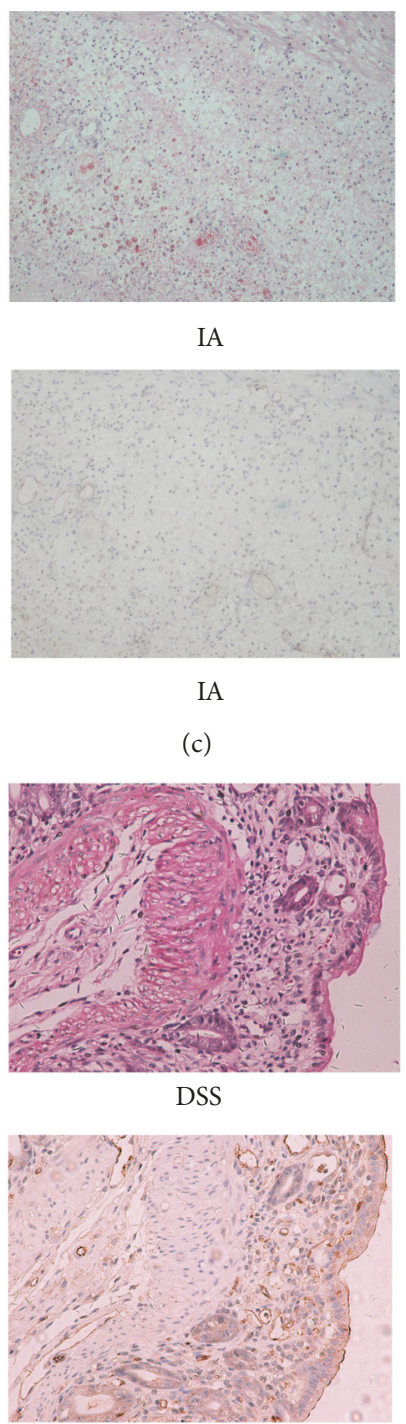

(d)

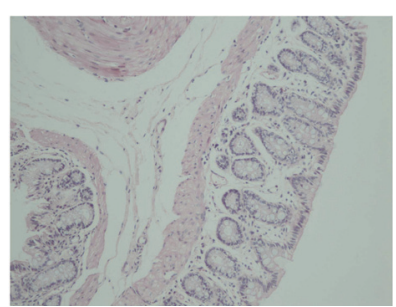

$\mathrm{IA}+\mathrm{PN}(1.0 \mathrm{~g} / \mathrm{kg})$

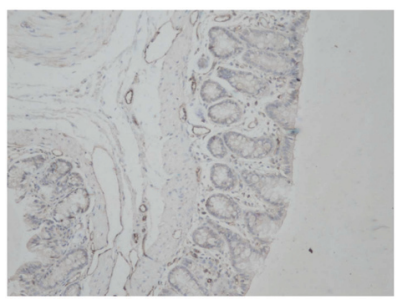

$\mathrm{IA}+\mathrm{PN}(1.0 \mathrm{~g} / \mathrm{kg})$

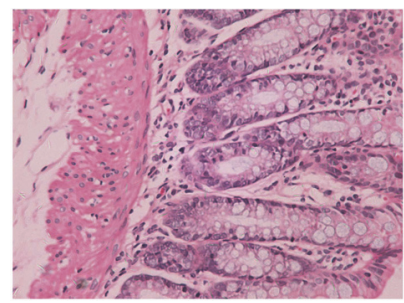

DSS + PN $(1.0 \mathrm{~g} / \mathrm{kg})$

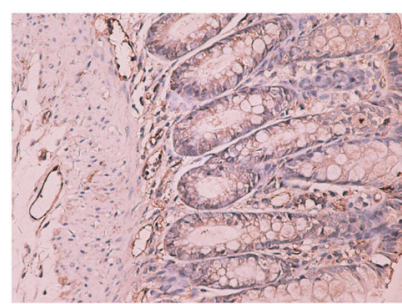

DSS + PN $(1.0 \mathrm{~g} / \mathrm{kg})$

Figure 2: Continued. 


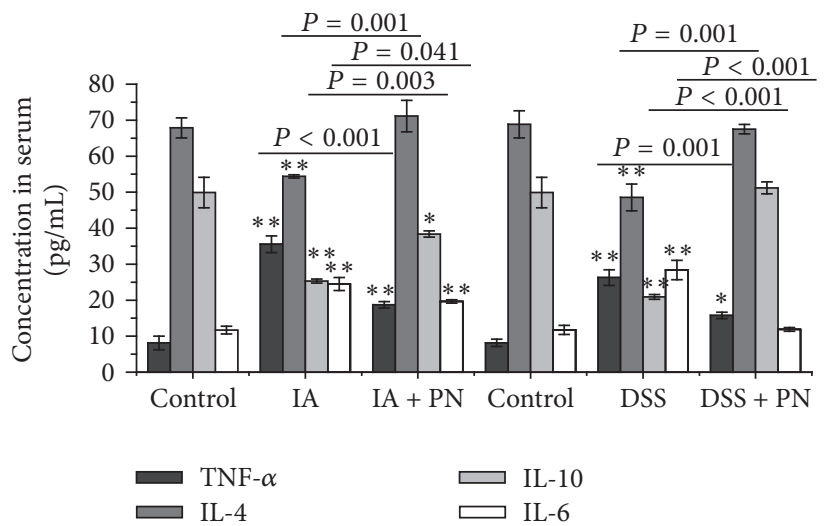

(e)

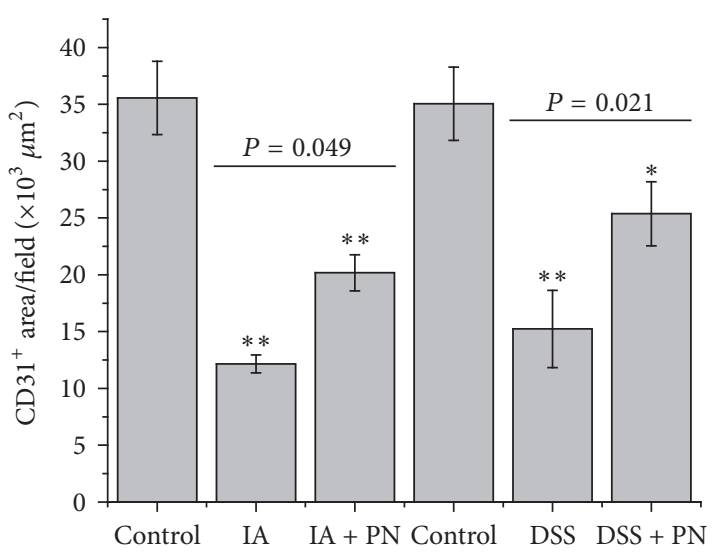

(f)

FIGURE 2: The efficacy of PN on colonic microvascular injury and mucosal damage. (a) Experimental induction of UC and treatment. (b) Profile of DAI, (c, d, f) histopathological examination of the colon (magnification, $\times 400$ ) and MVD, (e) serum levels of TNF- $\alpha$, IL-4, IL-6, and IL-10 in the study. All data, expressed as means \pm standard error of the mean (SEM) of three independent experiments performed in triplicate, were analyzed by one-way ANOVA and Student's $t$-test $\left({ }^{*} P<0.05,{ }^{* *} P<0.01\right)$.

and the function of microvessels in mucosa. When inflammation occurs in colonic mucosa, it causes increased oxygen consumption and malfunction of microvessels which results in decreased oxygen supply $[5,16]$. The imbalance of oxygen supply and oxygen consumption causes hypoxia and apoptosis of endothelium cells which aggravates the mucosa injury $[5,17]$. It was also found in the previous study that PN could promote repair of mucosal ulcer and reduce inflammatory response in patients with inflammatory bowel diseases (IBD) including ulcerative colitis (UC) and Crohn's disease (CD) [18]. This suggested that PN might repair mucosal ulcer via attenuating microvascular injury. Proangiogenic therapy has been considered as a new challenge for gastrointestinal ulcers [19].

However, the effect of $\mathrm{PN}$ on regulating the angiogenesis is still controversial. Qiao et al. found that PN extract could inhibit angiogenesis of atherosclerotic plaque by downregulating VEGF [20]. On the contrary, Yang et al. found PN had the effects of proangiogenesis and antiapoptosis [21]. Therefore, to further clarify the effect of $\mathrm{PN}$ on angiogenesis is critical to its clinical application. Our results showed that the degree of mucosal inflammation and damage was closely related to the degree of vascular injuries. After treatment by $\mathrm{PN}$, the microvascular injury and the mucosal damage were attenuated in the experimental colitis, which was similar to the previous study [8].

Although many putative cytokines have been considered as regulators of angiogenesis, VEGF, especially VEGFA, plays most important role in angiogenesis [22, 23]. Both VEGFA121 and VEGFA165, two isoforms of VEGF, are closely related to the development of colonic microvessels. We also found that the increased ratio of VEGFA165/VEGFA121 was correlated with the decreased numbers of microvessels [6]. In this study, increased VEGFA121 and VEGFA165 were downregulated by $\mathrm{PN}$ in rats with colitis accompanied with attenuation of microvascular injury.

In addition, our results showed in previous study that Rap1GAP/TSP signaling pathway was important in regulating the development of colonic microvessels [6]. The increasing expression of Rap1GAP and TSP1 proteins had a close relationship with the damage of mucosa and microvessels in colon. With the expression of Rap1GAP and TSP1 proteins inhibited, microvascular injury was decreased in colon. In the study, the expression of Rap1GAP and TSP1 proteins was significantly downregulated by $\mathrm{PN}$ in colonic mucosa with the microvascular injury decreased in the rats with colitis.

\section{Conclusions}

In summary, PN could downregulate increased VEGFA121 and VEGFA165 and block Rap1GAP/TSP1 signaling pathway in experimental colitis so that it attenuates microvascular injury, which resulted in improving repair of mucosal injury. There are still some issues unsolved in this study. How does PN regulate VEGFA121 and VEGFA165? Which component of $\mathrm{PN}$ plays the critical role in angiogenesis regulation? These are important for using PN to treat mucosal injury and need further exploring.
Abbreviations
PN: $\quad$ Panax notoginseng
UC: $\quad$ Ulcerative colitis
HPLC-DAD: High performance liquid chromatography-diode array detection
DSS: Dextran sodium sulfate
IA: Iodoacetamide
SD: $\quad$ Sprague-Dawley 


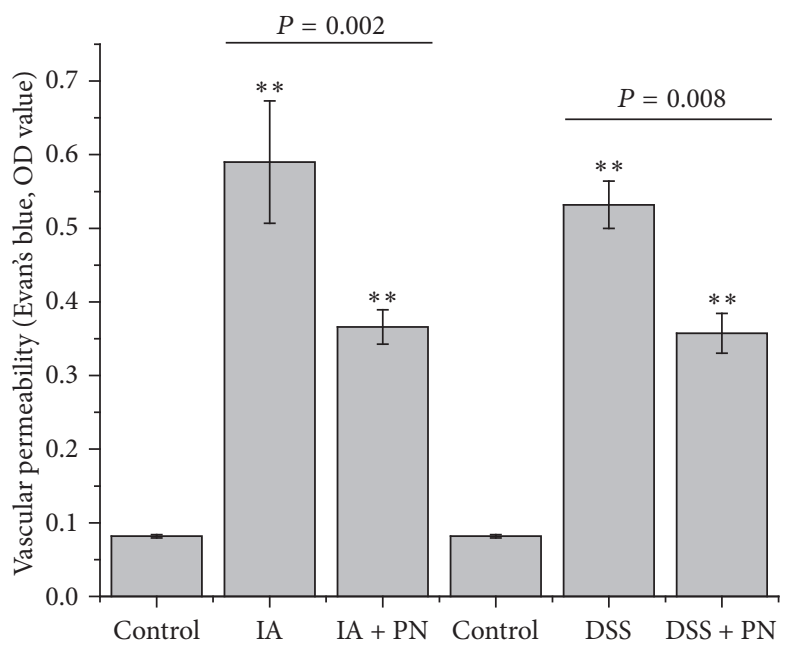

(a)

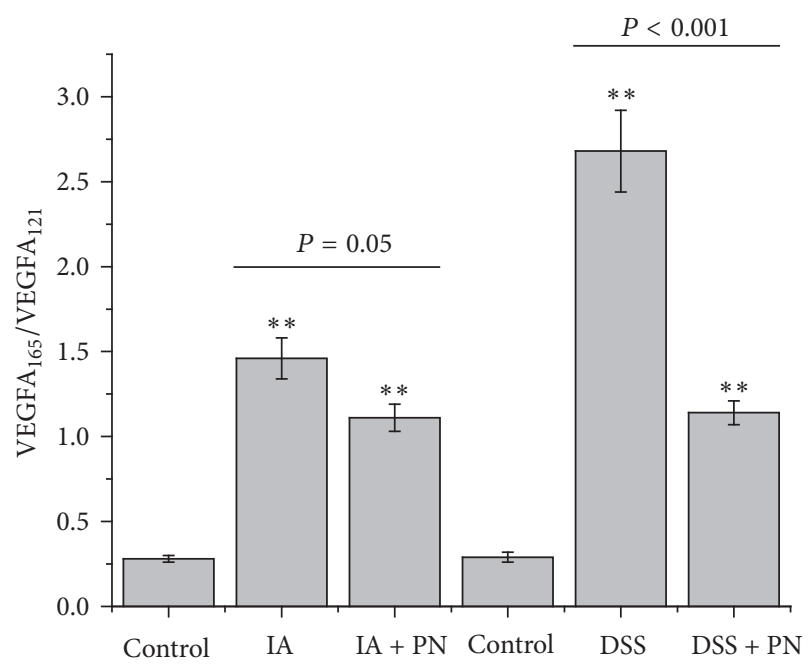

(b)

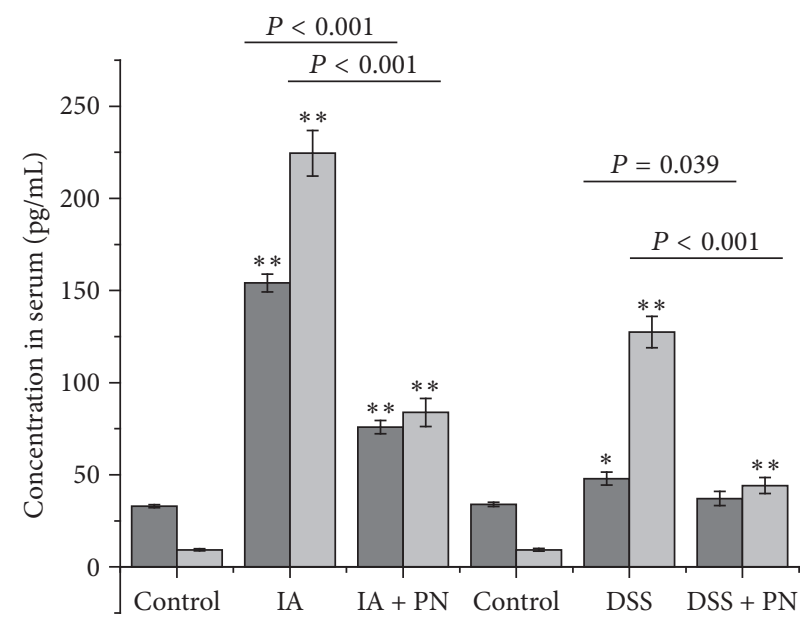

VEGFA121

VEGFA165

(c)

FIGURE 3: PN reversed the vascular permeability (VP) in colon and the disordered serum ratio of VEGFA165/VEGFA121. (a) Profile of VP, (b, c) serum levels of VEGFA165 and VEGFA121, and the ratio of VEGFA165/VEGFA121 in the study. All data, expressed as means \pm SEM of three independent experiments performed in triplicate, were analyzed by one-way ANOVA and Student's $t$-test $\left({ }^{*} P<0.05,{ }^{* *} P<0.01\right)$.

MVD: Microvessel density

VEGF: Vascular endothelial growth factor

IL: Interleukin.

\section{Conflicts of Interest}

The authors declared that no conflicts of interest existed.

\section{Authors' Contributions}

Shiying Wang and Ping Tao contributed equally to this work. Shiying Wang and Ping Tao performed experiments, analyzed data, prepared figures for the manuscript, and contributed to manuscript writing; Wangjun Zhang performed experiments and analyzed data; Lei Zhao provided research material; Jiang Lin and Hongyi Hu contributed to experimental design, data interpretation, manuscript writing, and project supervision. All authors approved the final version.

\section{Acknowledgments}

The authors thank all technical staffs who provided help in the study. This work was supported by National Natural Science Foundation of China (no. 81373616) and Shanghai 


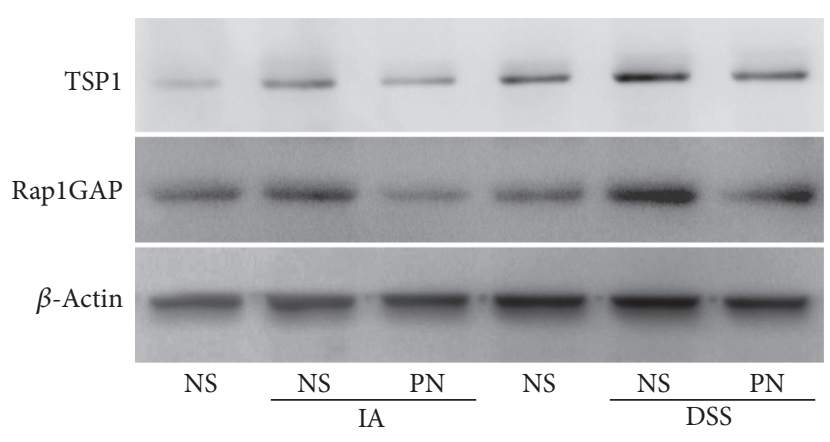

(a)

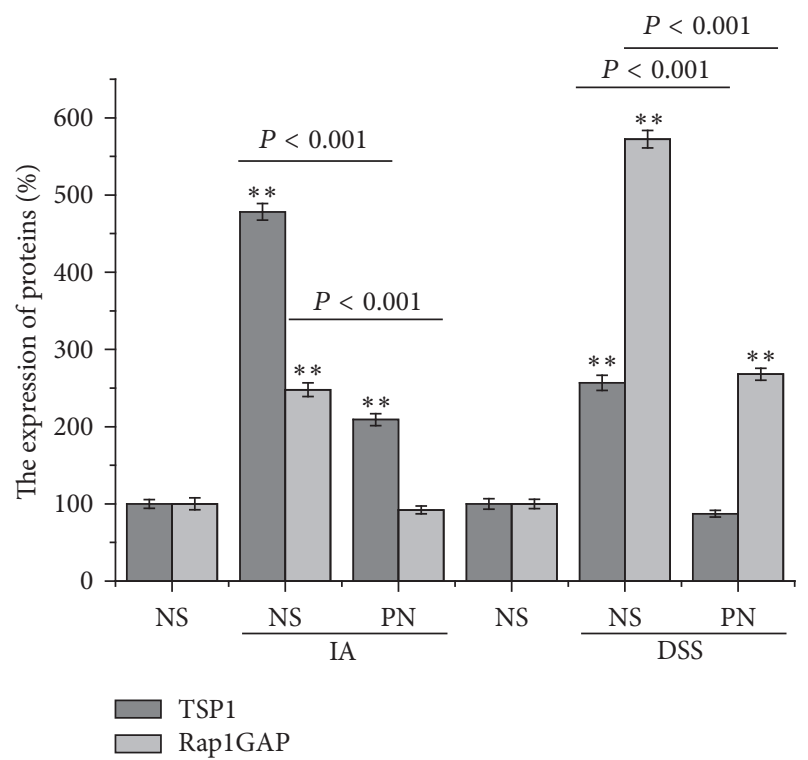

(b)

Figure 4: PN blocked Rap1GAP/TSP1 signaling pathway. (a, b) The expression level of Rap1GAP and TSP1 proteins in colonic mucosa in rats. All data, expressed as means \pm SEM of three independent experiments performed in triplicate, were analyzed by one-way ANOVA and Student's $t$-test $\left({ }^{* *} P<0.01\right)$.

TCM Development Committee (Research on the Academic School of TCM Master Huang Wendong).

\section{References}

[1] A. Kornbluth and D. B. Sachar, "Ulcerative colitis practice guidelines in adults: american college of gastroenterology, practice parameters committee," American Journal of Gastroenterology, vol. 105, no. 3, pp. 501-523, 2010.

[2] S. Netzel-Arnett, M. S. Buzza, T. Shea-Donohue et al., "Matriptase protects against experimental colitis and promotes intestinal barrier recovery," Inflammatory Bowel Diseases, vol. 18, no. 7, pp. 1303-1314, 2012.

[3] G. Tolstanova, X. Deng, S. W. French et al., "Early endothelial damage and increased colonic vascular permeability in the development of experimental ulcerative colitis in rats and mice," Laboratory Investigation, vol. 92, no. 1, pp. 9-21, 2012.

[4] J. Li, D. Kong, Q. Wang et al., "Niacin ameliorates ulcerative colitis via prostaglandin D2-mediated D prostanoid receptor 1 activation," EMBO Molecular Medicine, vol. 9, no. 5, pp. 571-588, 2017.

[5] S. Van Welden, M. De Vos, B. Wielockx et al., "Haematopoietic prolyl hydroxylase-1 deficiency promotes M2 macrophage polarization and is both necessary and sufficient to protect against experimental colitis," The Journal of Pathology, vol. 241, no. 4, pp. 547-558, 2017.

[6] W. Shiying, S. Boyun, Y. Jianye et al., "The different effects of VEGFA121 and VEGFA165 on regulating angiogenesis depend on phosphorylation sites of VEGFR2," Inflammatory Bowel Diseases, vol. 23, no. 4, pp. 603-616, 2017.

[7] Y. Lei, L.-L. Tao, and G.-L. Wang, "Effect of extracts from Panax ginseng, Panax notoginseng, and Ligusticum chuanxiong on vascular smooth muscle cells of aging and hypertension rats,"
Zhongguo Zhong Xi Yi Jie He Za Zhi Zhongguo Zhongxiyi Jiehe Zazhi, vol. 32, no. 10, pp. 1374-1379, 2012.

[8] X. Wen, C. Wang, C. Yu et al., "Panax notoginseng attenuates experimental colitis in the azoxymethane/dextran sulfate sodium mouse model," Phytotherapy Research, vol. 28, no. 6, pp. 892-898, 2014.

[9] W. L. Kar, L. P. Yuen, R. N. S. Wong, and A. S. T. Wong, "Ginsenoside-Rg1 induces vascular endothelial growth factor expression through the glucocorticoid receptor-related phosphatidylinositol 3-kinase/Akt and $\beta$-catenin/T-cell factor-dependent pathway in human endothelial cells," The Journal of Biological Chemistry, vol. 281, no. 47, pp. 36280-36288, 2006.

[10] B.-R. Yang, S.-J. Hong, S. M.-Y. Lee et al., "Pro-angiogenic activity of notoginsenoside R1 in human umbilical vein endothelial cells in vitro and in a chemical-induced blood vessel loss model of zebrafish in vivo," Chinese Journal of Integrative Medicine, vol. 22, no. 6, pp. 420-429, 2016.

[11] Y.-H. Hwang, D.-G. Kim, W. Li, H. J. Yang, N.-H. Yim, and J. Y. Ma, "Anti-inflammatory effects of Forsythia suspensa in dextran sulfate sodium-induced colitis," Journal of Ethnopharmacology, vol. 206, pp. 73-77, 2017.

[12] W.-J. Wu, R. Yan, T. Li, Y.-P. Li, R.-N. Zhou, and Y.-T. Wang, "Pharmacokinetic alterations of rhubarb anthraquinones in experimental colitis induced by dextran sulfate sodium in the rat," Journal of Ethnopharmacology, vol. 198, pp. 600-607, 2017.

[13] J. E. Ghia, P. Blennerhassett, H. Kumar-Ondiveeran, E. F. Verdu, and S. M. Collins, "The vagus nerve: a tonic inhibitory influence associated with inflammatory bowel disease in a murine model," Gastroenterology, vol. 131, no. 4, pp. 1122-1130, 2006.

[14] G. Tolstanova, T. Khomenko, X. Deng et al., "Neutralizing antivascular endothelial growth factor (VEGF) antibody reduces severity of experimental ulcerative colitis in rats: direct evidence for the pathogenic role of VEGF," The Journal of Pharmacology and Experimental Therapeutics, vol. 328, no. 3, pp. 749-757, 2009. 
[15] T. Wang, R. Guo, G. Zhou et al., “Traditional uses, botany, phytochemistry, pharmacology and toxicology of Panax notoginseng (Burk.) F. H. Chen: a review," Journal of Ethnopharmacology, vol. 188, pp. 234-258, 2016.

[16] E. P. Cummins and D. Crean, "Hypoxia and inflammatory bowel disease," Microbes and Infection, vol. 19, no. 3, pp. 210-221, 2017.

[17] H. Saijo, N. Tatsumi, S. Arihiro et al., "Microangiopathy triggers, and inducible nitric oxide synthase exacerbates dextran sulfate sodium-induced colitis," Laboratory Investigation, vol. 95, no. 7, pp. 728-748, 2015.

[18] J. K. Triantafillidis, A. Triantafyllidi, C. Vagianos, and A. Papalois, "Favorable results from the use of herbal and plant products in inflammatory bowel disease: evidence from experimental animal studies," Annals of Gastroenterology, vol. 29, no. 3, pp. 268-281, 2016.

[19] S. Szabo, X. Deng, G. Tolstanova et al., "Angiogenic and antiangiogenic therapy for gastrointestinal ulcers: New challenges for rational therapeutic predictions and drug design," Current Pharmaceutical Design, vol. 17, no. 16, pp. 1633-1642, 2011.

[20] Y. Qiao, P.-J. Zhang, X.-T. Lu et al., "Panax notoginseng saponins inhibits atherosclerotic plaque angiogenesis by downregulating vascular endothelial growth factor and nicotinamide adenine dinucleotide phosphate oxidase subunit 4 expression," Chinese Journal of Integrative Medicine, vol. 21, no. 4, pp. 259265, 2015.

[21] B. R. Yang, K. K. Cheung, X. Zhou et al., "Amelioration of acute myocardial infarction by saponins from flower buds of Panax notoginseng via pro-angiogenesis and anti-apoptosis," Journal of Ethnopharmacology, vol. 181, pp. 50-58, 2016.

[22] H. Hosseini, M. Rajabibazl, W. Ebrahimizadeh, and G. R. Dehbidi, "Inhibiting angiogenesis with human single-chain variable fragment antibody targeting VEGF," Microvascular Research, vol. 97, pp. 13-18, 2015.

[23] A. Rossi, S. Gauvrit, M. Marass, L. Pan, C. B. Moens, and D. Y. R. Stainier, "Regulation of Vegf signaling by natural and synthetic ligands," Blood, vol. 128, no. 19, pp. 2359-2366, 2016. 


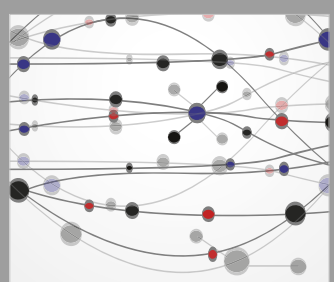

The Scientific World Journal
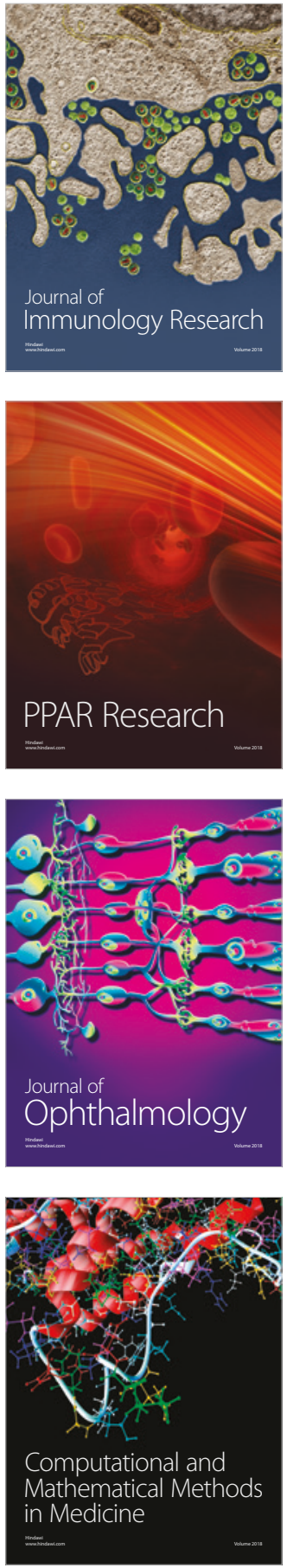

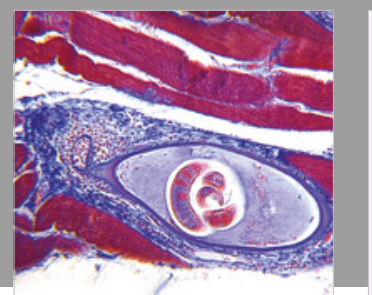

Gastroenterology Research and Practice

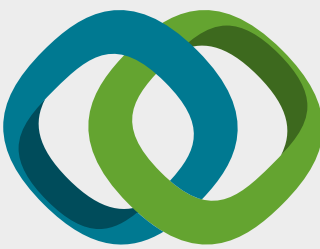

\section{Hindawi}

Submit your manuscripts at

www.hindawi.com
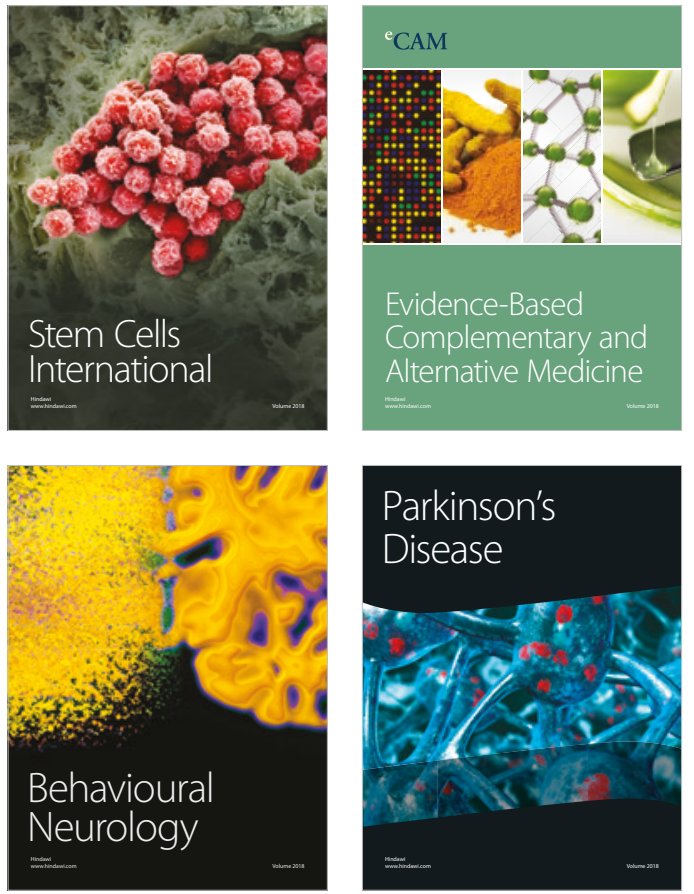

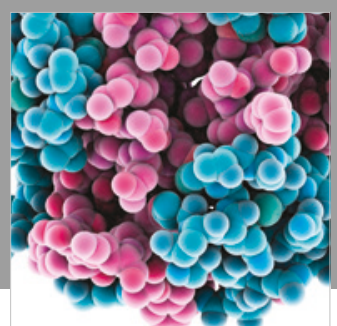

ournal of

Diabetes Research

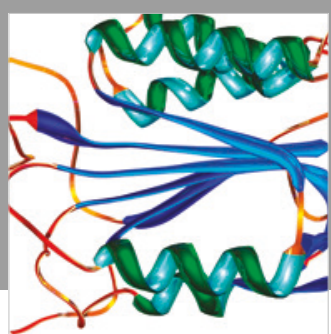

Disease Markers
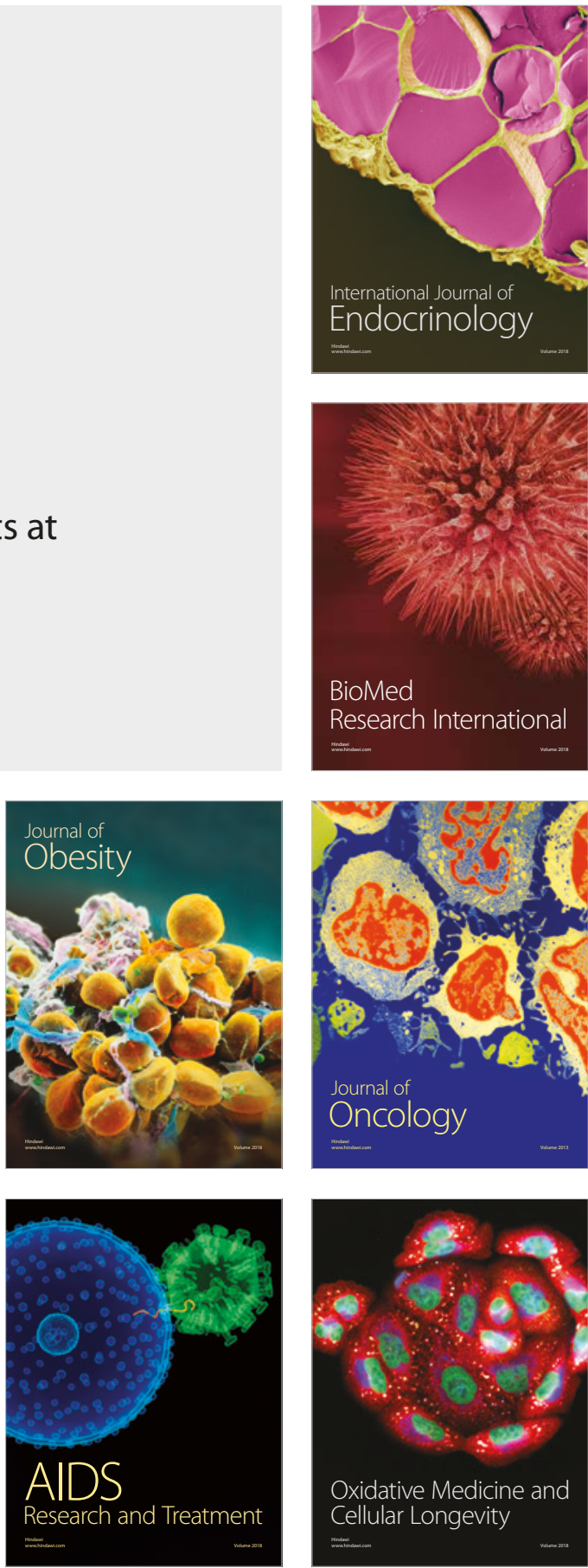